\title{
Article
}

\section{Pythagorean fuzzy multiset and its application to course placements}

\author{
Paul Augustine Ejegwa \\ Department of Mathematics/Statistics/Computer Science, University of Agriculture, P.M.B. 2373, Makurdi, Nigeria.; \\ ejegwa.augustine@uam.edu.ng; Tel.: +2347062583323
}

Received: 11 March 2020; Accepted: 1 April 2020; Published: 11 April 2020.

\begin{abstract}
The concept of fuzzy set theory is of paramount relevance to tackling the issues of uncertainties in real-life problems. In a quest to having a reasonable means of curbing imprecision, the idea of fuzzy sets had been generalized to intuitionistic fuzzy sets, fuzzy multisets, Pythagorean fuzzy sets among others. The notion of intuitionistic fuzzy multisets (IFMS) came into the limelight naturally because there are instances when repetitions of both membership and non-membership degrees cannot be ignored like in the treatment of patients, where each consultations are key in diagnosis and therapy. In IFMS theory, the sum of the degrees of membership and non-membership is less than or equals one at each levels. Supposing the sum of the degrees of membership and non-membership is greater than or equal to one at any level, then the concept of Pythagorean fuzzy multisets (PFMS) is appropriate to handling such scenario. In this paper, the idea of PFMS is proposed as an extensional Pythagorean fuzzy sets proposed by R. R. Yager. In fact, PFMS is a Pythagorean fuzzy set in the framework of multiset. The main objectives of this paper are to expatiate the operations under PFMSs and discuss some of their algebraic properties with some related results. The concepts of level sets, cuts, accuracy and score functions, and modal operators are established in the setting of PFMSs with a number of results. Finally, to demonstrate the applicability of the proposed soft computing technique, a course placements scenario is discussed via PFMS framework using composite relation defined on PFMSs. This soft computing technique could find expression in other multi-criteria decision-making (MCDM) problems.
\end{abstract}

Keywords: Course placement, fuzzy set, fuzzy multiset, intuitionistic fuzzy set, pythagorean fuzzy set, pythagorean fuzzy multiset.

MSC: 47S40, $03 E 72$.

\section{Introduction}

$\mathbf{F}$ uzzy set theory proposed by Zadeh [1] has achieved a huge impact in many fields to handle uncertainty/vagueness. Due to the vast majority of imprecise and vague information in real-life problems, different extensions of fuzzy set have been developed by some researchers. Yager [2] applied the idea of multiset [3], which is an extension of set with repeated elements in a collection to propose fuzzy multiset. Consequently, a fuzzy multiset allows repetition of membership degrees of elements in multiset framework. In fact, fuzzy multiset generalizes fuzzy set [4].

The concept of intuitionistic fuzzy sets (IFS) was proposed and studied in [5-7] as a generalization of fuzzy sets. The main advantage of the IFS is its ability to cope with the hesitancy that may exist due to information impression. This is achieved by incorporating a second function, along with the membership function, $\mu$ of the conventional fuzzy set, called non-membership function, $v$. The idea of IFS has found expression in many cases like medical diagnosis, career placements, pattern recognition and other MCDM problems [8-13].

However robust the notion of IFS is, there are circumstances where $\mu+v \geq 1$ unlike the situation captured in IFS (where, $\mu+v \leq 1$ ). The shortcoming in IFS naturally led to the introduction of a concept, called Pythagorean fuzzy sets (PFSs) by Yager [14]. PFS is a tool to deal with vagueness considering the membership grade, $\mu$ and non-membership grade, $v$ satisfying the condition $\mu+v \geq 1$. As a generalized set, PFS has close 
relationship with IFS. This idea can be used to characterize the uncertain information more sufficiently and accurately than IFS. PFSs have been applied in many areas, like the one discussed in [15].

In [16], the concepts of IFS and fuzzy multiset were combined to proposed intuitionistic fuzzy multisets (IFMS) as the generalization of IFS in multiset framework or the extension of fuzzy multisets by incorporating count non-membership functions, $C N=\left\{v^{1}, \ldots, v^{n}\right\}$ in addition to the count membership functions, $C M=$ $\left\{\mu^{1}, \ldots, \mu^{n}\right\}$ captured in fuzzy multisets. Some operations and modal operators on IFMS have been studied in $[17,18]$. Due to the resourcefulness of IFMS, it has been applied in many real-life problems as seen in [16-27].

The motivation of this paper follows from the ideas of PFSs [14] and IFMS [16]. The paper proposes Pythagorean fuzzy multisets (PFMSs), studies its properties and also, its application to course placements. PFMS is either the incorporation of IFMS in PFS setting or PFS in multiset framework.

The paper is organized by presenting some mathematical preliminaries such as fuzzy sets, fuzzy multisets, IFSs, IFMSs and PFSs in Section 2. Moreover, Section 3 covers the concept of PFMS and explicates the ideas of level sets, accuracy and score functions in the setting of PFMS. Also, the idea of cuts in PFMSs context is discussed with some results, and some modal operators on PFMSs are proposed with some deduced theorems. In Section 4, the application of PFMSs in course placements are discussed through composite relation defined on PFMSs. Finally, Section 5 summarises the paper and gives some useful conclusions.

\section{Preliminaries}

\subsection{Fuzzy sets}

Definition 1. [1] Let $X$ be a nonempty set. A fuzzy set $A$ of $X$ is characterized by a membership function

$$
\mu_{A}: X \rightarrow[0,1]
$$

That is,

$$
\mu_{A}(x)= \begin{cases}1, & \text { if } x \in X \\ 0, & \text { if } x \notin X \\ (0,1) & \text { if } x \text { is partly in } X\end{cases}
$$

Alternatively, a fuzzy set $A$ of $X$ is an object having the form

$$
A=\left\{\left\langle x, \mu_{A}(x)\right\rangle \mid x \in X\right\} \text { or } A=\left\{\left\langle\frac{\mu_{A}(x)}{x}\right\rangle \mid x \in X\right\},
$$

where the function

$$
\mu_{A}(x): X \rightarrow[0,1]
$$

defines the degree of membership of the element, $x \in X$.

\subsection{Fuzzy multisets}

Definition 2. [2] Assume $X$ is a set of elements. Then, a fuzzy bag/multiset $A$ drawn from $X$ can be characterized by a count membership function $C M_{A}$ such that

$$
C M_{A}: X \rightarrow Q,
$$

where $Q$ is the set of all crisp bags or multisets from the unit interval $I=[0,1]$.

A fuzzy multiset can also be characterized by a high-order function. In particular, a fuzzy multiset $A$ can be characterized by a function

$$
C M_{A}: X \rightarrow N^{I} \text { or } C M_{A}: X \rightarrow[0,1] \rightarrow N,
$$

where $I=[0,1]$ and $N=\mathbb{N} \cup\{0\}$.

It follows that $C M_{A}(x)$ for $x \in X$ is given as

$$
C M_{A}(x)=\left\{\mu_{A}^{1}(x), \mu_{A}^{2}(x), \ldots, \mu_{A}^{n}(x), \ldots\right\},
$$


where $\mu_{A}^{1}(x), \mu_{A}^{2}(x), \ldots, \mu_{A}^{n}(x), \ldots \in[0,1]$ such that $\mu_{A}^{1}(x) \geq \mu_{A}^{2}(x) \geq \ldots \geq \mu_{A}^{n}(x) \geq \ldots$, whereas in a finite case, we write

$$
C M_{A}(x)=\left\{\mu_{A}^{1}(x), \mu_{A}^{2}(x), \ldots, \mu_{A}^{n}(x)\right\},
$$

for $\mu_{A}^{1}(x) \geq \mu_{A}^{2}(x) \geq \ldots \geq \mu_{A}^{n}(x)$.

A fuzzy multiset $A$ can be represented in the form

$$
A=\left\{\left\langle\frac{C M_{A}(x)}{x}\right\rangle \mid x \in X\right\} \text { or } A=\left\{\left\langle x, C M_{A}(x)\right\rangle \mid x \in X\right\}
$$

\subsection{Intuitionistic fuzzy sets}

Definition 3. [5] Let a nonempty set $X$ be fixed. An IFS $A$ of $X$ is an object having the form

$$
A=\left\{\left\langle x, \mu_{A}(x), v_{A}(x)\right\rangle \mid x \in X\right\}
$$

or

$$
A=\left\{\left\langle\frac{\mu_{A}(x), v_{A}(x)}{x}\right\rangle \mid x \in X\right\}
$$

where the functions

$$
\mu_{A}(x): X \rightarrow[0,1] \text { and } v_{A}(x): X \rightarrow[0,1]
$$

define the degree of membership and the degree of non-membership, respectively of the element $x \in X$ to $A$, which is a subset of $X$, and for every $x \in X$,

$$
0 \leq \mu_{A}(x)+v_{A}(x) \leq 1 .
$$

For each $A$ in $X$,

$$
\pi_{A}(x)=1-\mu_{A}(x)-v_{A}(x)
$$

is the intuitionistic fuzzy set index or hesitation margin of $x$ in $X$. The hesitation margin $\pi_{A}(x)$ is the degree of non-determinacy of $x \in X$, to $A$ and $\pi_{A}(x) \in[0,1]$. The hesitation margin is the function that expresses lack of knowledge of whether $x \in X$ or $x \notin X$. Thus,

$$
\mu_{A}(x)+v_{A}(x)+\pi_{A}(x)=1 .
$$

\subsection{Intuitionistic fuzzy multisets}

Definition 4. [16] Let $X$ be a nonempty set. An IFMS $A$ drawn from $X$ is of the form

$$
A=\left\{\left\langle\frac{C M_{A}(x)}{x}, \frac{C N_{A}(x)}{x}\right\rangle \mid x \in X\right\}
$$

where

$$
C M_{A}(x)=\mu_{A}^{1}(x), \ldots, \mu_{A}^{n}(x), \ldots
$$

and

$$
C N_{A}(x)=v_{A}^{1}(x), \ldots, v_{A}^{n}(x), \ldots
$$

are the count membership and count non-membership degrees defined by the functions

$$
C M_{A}: X \rightarrow N^{[0,1]} \text { and } C N_{A}: X \rightarrow N^{[0,1]}
$$

such that $0 \leq C M_{A}(x)+C N_{A}(x) \leq 1$, where $N=\mathbb{N} \cup\{0\}$.

If the count membership functions and count non-membership functions have only $n$-terms (i.e. finite), then $n$ is called the dimension of $A$. Consequently 


$$
A=\left\{\left\langle\frac{\mu_{A}^{1}(x), \ldots, \mu_{A}^{n}(x)}{x}, \frac{v_{A}^{1}(x), \ldots, v_{A}^{n}(x)}{x}\right\rangle \mid x \in X\right\}
$$

for $i=1, \ldots, n$.

For each IFMS $A$ of $X$,

$$
\mathrm{CH}_{A}(x)=1-\mathrm{CM}_{A}(x)-\mathrm{CN}_{A}(x)
$$

is the intuitionistic fuzzy multisets index or count hesitation margin of $x$ in $A$, where

$$
C H_{A}(x)=\pi_{A}^{1}(x), \ldots, \pi_{A}^{n} \text {. }
$$

The hesitation margin $\pi_{A}^{i}(x)$ for each $i=1, \ldots, n$ is the degree of non-determinacy of $x \in X$ to $A$ and $\pi_{A}^{i}(x) \in[0,1]$. The count hesitation margin is the function that expresses lack of knowledge of whether $x \in A$ or $x \notin A$. Thus,

$$
\mu_{A}^{i}(x)+v_{A}^{i}(x)+\pi_{A}^{i}(x)=1
$$

for each $i=1, \ldots, n$.

\subsection{Pythagorean fuzzy sets}

Definition 5. [14] Let $X$ be a universal set. Then, an PFS $A$ of $X$ is a set of ordered pairs defined by

$$
A=\left\{\left\langle x, \mu_{A}(x), v_{A}(x)\right\rangle \mid x \in X\right\}
$$

or

$$
A=\left\{\left\langle\frac{\mu_{A}(x), v_{A}(x)}{x}\right\rangle \mid x \in X\right\}
$$

where the functions

$$
\mu_{A}(x): X \rightarrow[0,1] \text { and } v_{A}(x): X \rightarrow[0,1]
$$

define the degree of membership and the degree of non-membership, respectively of the element $x \in X$ to $A$, which is a subset of $X$, and for every $x \in X$,

$$
0 \leq\left(\mu_{A}(x)\right)^{2}+\left(v_{A}(x)\right)^{2} \leq 1 .
$$

Supposing $\left(\mu_{A}(x)\right)^{2}+\left(v_{A}(x)\right)^{2} \leq 1$, then there is a degree of indeterminacy of $x \in X$ to $A$ defined by $\pi_{A}(x)=\sqrt{1-\left[\left(\mu_{A}(x)\right)^{2}+\left(v_{A}(x)\right)^{2}\right]}$ and $\pi_{A}(x) \in[0,1]$. In what follows, $\left(\mu_{A}(x)\right)^{2}+\left(v_{A}(x)\right)^{2}+\left(\pi_{A}(x)\right)^{2}=$ 1. Otherwise, $\pi_{A}(x)=0$ whenever $\left(\mu_{A}(x)\right)^{2}+\left(v_{A}(x)\right)^{2}=1$.

\section{Pythagorean fuzzy multisets}

Definition 6. Let $X$ be a nonempty set. Then, an PFMS $A$ drawn from $X$ is of the form

$$
A=\left\{\left\langle\frac{C M_{A}(x)}{x}, \frac{C N_{A}(x)}{x}\right\rangle \mid x \in X\right\}
$$

or

$$
A=\left\{\left\langle x, C M_{A}(x), C N_{A}(x)\right\rangle \mid x \in X\right\}
$$

where

$$
C M_{A}(x)=\mu_{A}^{1}(x), \ldots, \mu_{A}^{n}(x)
$$

and

$$
C N_{A}(x)=v_{A}^{1}(x), \ldots, v_{A}^{n}(x)
$$

are the count membership and count non-membership degrees defined by the functions

$$
C M_{A}: X \rightarrow N^{[0,1]} \text { and } C N_{A}: X \rightarrow N^{[0,1]}
$$


such that $0 \leq\left[C M_{A}(x)\right]^{2}+\left[C N_{A}(x)\right]^{2} \leq 1$, where $N=\mathbb{N} \cup\{0\}$.

For each PFMS $A$ of $X$,

$$
C H_{A}(x)=\sqrt{1-\left[C M_{A}(x)\right]^{2}-\left[C N_{A}(x)\right]^{2}}
$$

is the count hesitation margin of $x$ in $A$, where

$$
\mathrm{CH}_{A}(x)=\pi_{A}^{1}(x), \ldots, \pi_{A}^{n} .
$$

The count hesitation margin $\mathrm{CH}_{A}(x)$ is the degree of non-determinacy of $x \in X$ to $A$ and $C H_{A}(x) \in[0,1]$. The count hesitation margin is the function that expresses lack of knowledge of whether $x \in A$ or $x \notin A$. Thus,

$$
\left[\mathrm{CM}_{A}(x)\right]^{2}+\left[\mathrm{CN}_{A}(x)\right]^{2}+\left[\mathrm{CH}_{A}(x)\right]^{2}=1 .
$$

We denote the set of all PFMS over $X$ by PFMS $(X)$. Table 1 explains the difference between IFMS and PFMS.

Table 1. IFMS and PFMS

\begin{tabular}{||c|c||}
\hline \hline IFMS & PFMS \\
\hline \hline$C M+C N \leq 1$ & $C M+C N \leq 1$ or $C M+C N \geq 1$ \\
$0 \leq C M+C N \leq 1$ & $0 \leq C M^{2}+C N^{2} \leq 1$ \\
$C H=1-(C M+C N)$ & $C H=\sqrt{1-\left[C M^{2}+C N^{2}\right]}$ \\
$C M+C N+C H=1$ & $C M^{2}+C N^{2}+C H^{2}=1$ \\
\hline \hline
\end{tabular}

Example 1. Let $A$ be an PFMS of $X=\{x, y\}$ such that

$$
\begin{aligned}
& C M_{A}(x)=0.7,0.5,0.4 \\
& C N_{A}(x)=0.3,0.5,0.6 \\
& C M_{A}(y)=0.8,0.6,0.4 \\
& C N_{A}(y)=0.4,0.5,0.5 .
\end{aligned}
$$

That is

$$
A=\left\{\frac{\langle 0.7,0.5,0.4\rangle,\langle 0.3,0.5,0.6\rangle}{x}, \frac{\langle 0.8,0.6,0.4\rangle,\langle 0.4,0.5,0.5\rangle}{y}\right\} .
$$

Then

$$
\begin{aligned}
& \mathrm{CH}_{A}(x)=0.6481,0.7071,0.6928 \\
& \mathrm{CH}_{A}(y)=0.4472,0.6245,0.7681 .
\end{aligned}
$$

For easy computational purpose, an PFMS can be converted to PFS by taking the mean values of the count membership degrees, count non-membership degrees and count hesitation margin, respectively. That is, an PFMS $A$ in Example 1 becomes an PFS

$$
A=\left\{\frac{\langle 0.5333,0.4667\rangle}{x}, \frac{\langle 0.6,0.4667\rangle}{y}\right\} .
$$

Definition 7. Two PFMSs $A$ and $B$ are said to be equal or comparable if

$$
C M_{A}(x)=C M_{B}(x), C N_{A}(x)=C N_{B}(x)
$$

$\forall x \in X$ 
Definition 8. Let $A, B \in \operatorname{PFMS}(X)$, then $A$ is contained in $B$ denoted by $A \subseteq B$ if

$$
C M_{A}(x) \leq C M_{B}(x) \text { and } C N_{A}(x) \geq C N_{B}(x) \forall x \in X .
$$

We say $A$ is properly contained in $B$, that is, $A \subset B$ if $A \subseteq B$ and $A \neq B$. It means $C M_{A}(x) \leq C M_{B}(x)$ and $C N_{A}(x) \geq C N_{B}(x)$ but $C M_{A}(x) \neq C M_{B}(x)$ and $C N_{A}(x) \neq C N_{B}(x) \forall x \in X$.

Definition 9. Let $X$ and $Y$ be nonempty sets and let $f: X \rightarrow Y$ be a mapping. Suppose $A \in P F M S(X)$ and $B \in \operatorname{PFMS}(Y)$, respectively. Then

(i) the inverse image of $B$ under $f$, denoted by $f^{-1}(B)$, is an PFMS of $X$ defined by

$$
f^{-1}(B)=\left\{\left\langle\frac{C M_{f^{-1}(B)}(x)}{x}, \frac{C N_{f^{-1}(B)}(x)}{x}\right\rangle \mid x \in X\right\},
$$

where $C M_{f^{-1}(B)}(x)=C M_{B}(f(x))$ and $C N_{f^{-1}(B)}(x)=C N_{B}(f(x))$

$\forall x \in X$.

(ii) the image of $A$ under $f$, denoted by $f(A)$, is an PFMS of $Y$ defined by

$$
f(A)=\left\{\left\langle\frac{C M_{f(A)}(y)}{y}, \frac{C N_{f(A)}(y)}{y}\right\rangle \mid y \in Y\right\},
$$

where

$$
C M_{f(A)}(y)= \begin{cases}\bigvee_{x \in f^{-1}(y)} C M_{A}(x), & f^{-1}(y) \neq \varnothing \\ 0, & \text { otherwise }\end{cases}
$$

and

$$
C N_{f(A)}(y)= \begin{cases}\bigwedge_{x \in f^{-1}(y)} C N_{A}(x), & f^{-1}(y) \neq \varnothing \\ 0, & \text { otherwise }\end{cases}
$$

for each $y \in Y$. This is called the extension principle of PFMS.

Theorem 10. Let $A \in \operatorname{PFMS}(X)$. Suppose that $\mathrm{CH}_{A}(x)=0$, then the following hold:

(i) $\left|C M_{A}(x)\right|=\sqrt{\left|\left(C N_{A}(x)+1\right)\left(C N_{A}(x)-1\right)\right|}$.

(ii) $\left|C N_{A}(x)\right|=\sqrt{\left|\left(C M_{A}(x)+1\right)\left(C M_{A}(x)-1\right)\right|}$.

Proof. Suppose $x \in X$ and $A \in \operatorname{PFMS}(X)$. Then we prove (i) and (ii). Since $C H_{A}(x)=0$ for every $x \in X$, we have

$$
\begin{aligned}
& \left(C M_{A}(x)\right)^{2}+\left(C N_{A}(x)\right)^{2}=1 \\
& \Rightarrow-\left(C M_{A}(x)\right)^{2}=\left(C N_{A}(x)\right)^{2}-1
\end{aligned}
$$$$
\Rightarrow-\left(C M_{A}(x)\right)^{2}=\left(C N_{A}(x)+1\right)\left(C N_{A}(x)-1\right)
$$$$
\Rightarrow\left|\left(C M_{A}(x)\right)^{2}\right|=\left|\left(C N_{A}(x)+1\right)\left(C N_{A}(x)-1\right)\right|
$$$$
\Rightarrow\left|C M_{A}(x)\right|^{2}=\left|\left(C N_{A}(x)+1\right)\left(C N_{A}(x)-1\right)\right|
$$$$
\Rightarrow\left|C M_{A}(x)\right|=\sqrt{\left|\left(C N_{A}(x)+1\right)\left(C N_{A}(x)-1\right)\right|},
$$

which proves $(i)$. The proof of $(i i)$ is similar to that of $(i)$.

\subsection{Some operations under PFMSs}

Definition 11. For any two PFMSs $A$ and $B$ drawn from $X$, the following operations hold.

(i) Complement

$$
A^{c}=\left\{\left\langle\frac{C N_{A}(x)}{x}, \frac{C M_{A}(x)}{x}\right\rangle \mid x \in X\right\}
$$

(ii) Union

$$
A \cup B=\left\{\left\langle\frac{\max \left(C M_{A}(x), C M_{B}(x)\right)}{x}, \frac{\min \left(C N_{A}(x), C N_{B}(x)\right)}{x}\right\rangle \mid x \in X\right\}
$$


(iii) Intersection

$$
A \cup B=\left\{\left\langle\frac{\min \left(C M_{A}(x), C M_{B}(x)\right)}{x}, \frac{\max \left(C N_{A}(x), C N_{B}(x)\right)}{x}\right\rangle \mid x \in X\right\} .
$$

Definition 12. Let $A, B \in \operatorname{PFMS}(X)$. Then, the addition of $A$ and $B$ is defined as

$$
A \oplus B=\left\{\left\langle\frac{\sqrt{\left(C M_{A}(x)\right)^{2}+\left(C M_{B}(x)\right)^{2}-\left(C M_{A}(x)\right)^{2}\left(C M_{B}(x)\right)^{2}},}{x}, \frac{C N_{A}(x) C N_{B}(x)}{x}\right\rangle \mid x \in X\right\},
$$

and the multiplication of $A$ and $B$ is defined as

$$
A \otimes B=\left\{\left\langle\frac{C M_{A}(x) C M_{B}(x)}{x}, \frac{\sqrt{\left(C N_{A}(x)\right)^{2}+\left(C N_{B}(x)\right)^{2}-\left(C N_{A}(x)\right)^{2}\left(C N_{B}(x)\right)^{2}}}{x}\right\rangle \mid x \in X\right\} .
$$

Proposition 1. Let $A, B, C \in P F M S(X)$, then the following properties follow.

(i) Complementary law

$$
\left(A^{c}\right)^{c}=A
$$

(ii) Idempotent laws

$$
\begin{aligned}
& A \cup A=A \\
& A \cap A=A
\end{aligned}
$$

(iii) Commutative laws

$$
\begin{aligned}
& A \cup B=B \cup A \\
& A \cap B=B \cap A \\
& A \oplus B=B \oplus A \\
& A \otimes B=B \otimes A
\end{aligned}
$$

(iv) Associative laws

$$
\begin{aligned}
& (A \cup B) \cup C=A \cup(B \cup C) \\
& (A \cap B) \cap C=A \cap(B \cap C) \\
& (A \oplus B) \oplus C=A \oplus(B \oplus C) \\
& (A \otimes B) \otimes C=A \otimes(B \otimes C)
\end{aligned}
$$

(v) Distributive laws

$$
\begin{aligned}
& A \cup(B \cap C)=(A \cup B) \cap(A \cup C) \\
& A \cap(B \cup C)=(A \cap B) \cup(A \cap C) \\
& A \oplus(B \cup C)=(A \oplus B) \cup(A \oplus C) \\
& A \oplus(B \cap C)=(A \oplus B) \cap(A \oplus C) \\
& A \otimes(B \cup C)=(A \otimes B) \cup(A \otimes C) \\
& A \otimes(B \cap C)=(A \otimes B) \cap(A \otimes C)
\end{aligned}
$$

Distributive laws hold for both sides (right and left).

(vi) DeMorgan laws

$$
\begin{aligned}
& (A \cup B)^{c}=A^{c} \cap B^{c} \\
& (A \cap B)^{c}=A^{c} \cup B^{c} \\
& (A \oplus B)^{c}=A^{c} \otimes B^{c} \\
& (A \otimes B)^{c}=A^{c} \oplus B^{c}
\end{aligned}
$$


(vii) Absorption laws

$$
\begin{aligned}
& A \cap(A \cup B)=A \\
& A \cup(A \cap B)=A
\end{aligned}
$$

Proof. Straightforward, so we omit.

Theorem 13. Let $A, B \in \operatorname{PFMS}(X)$ such that $A=B^{c}$ and $B=A^{c}$, then

(i) $\left(A^{c} \cup B\right) \cap\left(A \cup B^{c}\right)=\left(A^{c} \cap B^{c}\right) \cup(A \cap B)$,

(ii) $\left(A^{\mathcal{C} \cap B}\right) \cup\left(A \cap B^{c}\right)=\left(A^{c} \cup B^{c}\right) \cap(A \cup B)$.

Proof. Since $A=B^{c}$ and $B=A^{c}$, we show that the left hand side (LHS) is equal to the right hand side (RHS). Now,

$$
\begin{aligned}
\left(A^{c} \cup B\right) \cap\left(A \cup B^{c}\right) & =(B \cup B) \cap(A \cup A) \\
& =A \cap B .
\end{aligned}
$$

Similarly,

$$
\begin{aligned}
\left(A^{c} \cap B^{c}\right) \cup(A \cap B) & =(B \cap A) \cup(A \cap B) \\
& =A \cap B .
\end{aligned}
$$

Thus, LHS=RHS, and hence $(i)$ is proved. The proof of $(i i)$ is similar to $(i)$, so we omit.

Definition 14. Let $A \in \operatorname{PFMS}(X)$. Then, the level/ground set of $A$ is defined by

$$
A_{*}=\left\{x \in X \mid C M_{A}(x)>0, C N_{A}(x)<1\right\} .
$$

Certainly, $A_{*}$ is a subset of $X$.

Proposition 2. Suppose $A$ and $B$ are PFMSs of a non-empty set $X$, then

(i) $(A \cap B)_{*}=A_{*} \cap B_{*}$,

(ii) $(A \cup B)_{*}=A_{*} \cup B_{*}$.

Proof. Straightforward, so we omit.

\subsection{Accuracy and score functions of PFMS}

Definition 15. Let $A \in \operatorname{PFMS}(X)$. Then the score function, $s$ of $A$ is defined by $s(A)=\Sigma_{i=1}^{n}\left[\left(C M_{A}\left(x_{i}\right)\right)^{2}-\right.$ $\left.\left(C N_{A}\left(x_{i}\right)\right)^{2}\right]$, where $s(A) \in[-1,1]$.

Definition 16. Let $A \in P F M S(X)$. Then the accuracy function, $a$ of $A$ is defined by $a(A)=\sum_{i=1}^{n}\left[\left(C M_{A}\left(x_{i}\right)\right)^{2}+\right.$ $\left.\left(C N_{A}\left(x_{i}\right)\right)^{2}\right]$ for $a(A) \in[0,1]$.

Theorem 17. Let $A \in P F M S(X)$. Then the following hold $\forall x \in X$ :

(i) $s(A)=0 \Leftrightarrow C M_{A}\left(x_{i}\right)=C N_{A}\left(x_{i}\right)$.

(ii) $s(A)=1 \Leftrightarrow\left|C N_{A}\left(x_{i}\right)\right|=\sqrt{\left|\left(1+C M_{A}\left(x_{i}\right)\right)\left(1-C M_{A}\left(x_{i}\right)\right)\right|}$.

(iii) $s(A)=-1 \Leftrightarrow C M_{A}\left(x_{i}\right)=\sqrt{\left(C N_{A}\left(x_{i}\right)+1\right)\left(C N_{A}\left(x_{i}\right)-1\right)}$.

Proof. (i) Suppose $s(A)=0$. Then $\left(C M_{A}\left(x_{i}\right)\right)^{2}=\left(C N_{A}\left(x_{i}\right)\right)^{2}$ implies $C M_{A}\left(x_{i}\right)=C N_{A}\left(x_{i}\right) \forall x_{i} \in X$. Conversely, assume $C M_{A}\left(x_{i}\right)=C N_{A}\left(x_{i}\right)$. Then $\left(C M_{A}\left(x_{i}\right)\right)^{2}=\left(C N_{A}\left(x_{i}\right)\right)^{2} \forall x_{i} \in X$. Thus $\left(C M_{A}\left(x_{i}\right)\right)^{2}-$ $\left(C N_{A}\left(x_{i}\right)\right)^{2}=0$. Hence $s(A)=0$. 
(ii) Suppose $s(A)=1$. Then

$1-\left(C M_{A}\left(x_{i}\right)\right)^{2}=-\left(C N_{A}\left(x_{i}\right)\right)^{2}$

$\Rightarrow\left(1+C M_{A}\left(x_{i}\right)\right)\left(1-C M_{A}\left(x_{i}\right)\right)=-\left(C N_{A}\left(x_{i}\right)\right)^{2}$

$\Rightarrow\left|\left(1+C M_{A}\left(x_{i}\right)\right)\left(1-C M_{A}\left(x_{i}\right)\right)\right|=\left|C N_{A}\left(x_{i}\right)\right|^{2}$

$\Rightarrow\left|C N_{A}\left(x_{i}\right)\right|=\sqrt{\left|\left(1+C M_{A}\left(x_{i}\right)\right)\left(1-C M_{A}\left(x_{i}\right)\right)\right|} \forall x_{i} \in X$.

Conversely, assume $\left|C N_{A}\left(x_{i}\right)\right|=\sqrt{\left|\left(1+C M_{A}\left(x_{i}\right)\right)\left(1-C M_{A}\left(x_{i}\right)\right)\right|}$. So we get

$\left|C N_{A}\left(x_{i}\right)\right|^{2}=\sqrt{\left|1-\left(C M_{A}\left(x_{i}\right)\right)^{2}\right|}$

$\Rightarrow\left(C N_{A}\left(x_{i}\right)\right)^{2}=1-\left(C M_{A}\left(x_{i}\right)\right)^{2}$ or $\left|C N_{A}\left(x_{i}\right)\right|^{2}=\sqrt{\left|1-\left(C M_{A}\left(x_{i}\right)\right)^{2}\right|}$

$\Rightarrow-\left(C N_{A}\left(x_{i}\right)\right)^{2}=1-\left(C M_{A}\left(x_{i}\right)\right)^{2} . \quad$ Take $-\left(C N_{A}\left(x_{i}\right)\right)^{2}=1-\left(C M_{A}\left(x_{i}\right)\right)^{2} \Rightarrow\left(C M_{A}\left(x_{i}\right)\right)^{2}-$ $\left(C N_{A}\left(x_{i}\right)\right)^{2}=1 \Rightarrow s(A)=1$.

(iii) Suppose $s(A)=-1$. Then

$\left(C N_{A}\left(x_{i}\right)\right)^{2}-1=\left(C M_{A}\left(x_{i}\right)\right)^{2}$

$\Rightarrow\left(C N_{A}\left(x_{i}\right)-1\right)\left(C N_{A}\left(x_{i}\right)+1\right)=\left(C M_{A}\left(x_{i}\right)\right)^{2}$

$\Rightarrow C M_{A}\left(x_{i}\right)=\sqrt{\left(C N_{A}\left(x_{i}\right)-1\right)\left(C N_{A}\left(x_{i}\right)+1\right)}$.

Conversely, suppose $C M_{A}\left(x_{i}\right)=\sqrt{\left(C N_{A}\left(x_{i}\right)-1\right)\left(C N_{A}\left(x_{i}\right)+1\right)}$. Then

$\left(C M_{A}\left(x_{i}\right)\right)^{2}=\left(C N_{A}\left(x_{i}\right)\right)^{2}-1 \Rightarrow\left(C M_{A}\left(x_{i}\right)\right)^{2}-\left(C N_{A}\left(x_{i}\right)\right)^{2}=-1 \Rightarrow s(A)=-1$.

Theorem 18. Let $A \in P F M S(X)$. Then the following statements hold $\forall x \in X$ :

(i) $a(A)=1 \Leftrightarrow \mathrm{CH}_{A}\left(x_{i}\right)=0$.

(ii) $a(A)=0 \Leftrightarrow\left|C M_{A}\left(x_{i}\right)\right|=\left|C N_{A}\left(x_{i}\right)\right|$.

Proof. (i) Suppose $a(A)=1$. So we have $\left(C M_{A}\left(x_{i}\right)\right)^{2}+\left(C N_{A}\left(x_{i}\right)\right)^{2}=1$, that is, $C H_{A}\left(x_{i}\right)=0$ since $\mathrm{CH}_{A}\left(x_{i}\right)=\sqrt{1-\left[\left(C M_{A}\left(x_{i}\right)\right)^{2}+\left(C N_{A}\left(x_{i}\right)\right)^{2}\right]}$.

Conversely, assume that $\mathrm{CH}_{A}\left(x_{i}\right)=0$. Then it follows that

$$
\left(C M_{A}\left(x_{i}\right)\right)^{2}+\left(C N_{A}\left(x_{i}\right)\right)^{2}=1 \Rightarrow a(A)=1 .
$$

(ii) Suppose $a(A)=0$. Then $\left(C M_{A}\left(x_{i}\right)\right)^{2}=-\left(C N_{A}\left(x_{i}\right)\right)^{2}$ or $\left(C N_{A}\left(x_{i}\right)\right)^{2}=-\left(C M_{A}\left(x_{i}\right)\right)^{2} \Leftrightarrow\left|C M_{A}\left(x_{i}\right)\right|^{2}=$ $\left|C N_{A}\left(x_{i}\right)\right|^{2} \Leftrightarrow\left|C M_{A}\left(x_{i}\right)\right|=\left|C N_{A}\left(x_{i}\right)\right|$.

\subsection{Some properties of PFMSs}

\subsection{1. $(\alpha, \beta)$-cuts of PFMS}

Definition 19. Let $A \in \operatorname{PFMS}(X)$. Then for $\alpha, \beta \in[0,1]$, the sets $A_{[\alpha, \beta]}$ and $A_{(\alpha, \beta)}$ defined by

$$
A_{[\alpha, \beta]}=\left\{x \in X \mid C M_{A}(x) \geq \alpha, C N_{A}(x) \leq \beta\right\}
$$

and

$$
A_{(\alpha, \beta)}=\left\{x \in X \mid C M_{A}(x)>\alpha, C N_{A}(x)<\beta\right\}
$$

are called strong and weak upper $\alpha, \beta$-cuts of $A$.

Similarly, the sets $A^{[\alpha, \beta]}$ and $A^{(\alpha, \beta)}$ defined by

$$
A^{[\alpha, \beta]}=\left\{x \in X \mid C M_{A}(x) \leq \alpha, C N_{A}(x) \geq \beta\right\}
$$

and

$$
A^{(\alpha, \beta)}=\left\{x \in X \mid C M_{A}(x)<\alpha, C N_{A}(x)>\beta\right\}
$$

are called strong and weak lower $\alpha, \beta$-cuts of $A$.

Remark 1. Let $A \in \operatorname{PFMS}(X)$ and take any $\alpha, \beta \in[0,1]$ such that $A_{[\alpha, \beta]}$ and $A^{[\alpha, \beta]}$ exist. Then, it follows that 
(i) $A_{(\alpha, \beta)} \subseteq A_{[\alpha, \beta]}$ and $A^{(\alpha, \beta)} \subseteq A^{[\alpha, \beta]}$.

(ii) $A_{[\alpha, \beta]}=B_{[\alpha, \beta]}, A_{(\alpha, \beta)}=B_{(\alpha, \beta)}, A^{[\alpha, \beta]}=B^{[\alpha, \beta]}$ and $A^{(\alpha, \beta)}=B^{(\alpha, \beta)}$ iff $A=B$.

For the purpose of this work, we shall be restricted to strong cuts of PFMS since $A_{(\alpha, \beta)} \subseteq A_{[\alpha, \beta]}$ and $A^{(\alpha, \beta)} \subseteq A^{[\alpha, \beta]}$.

Proposition 3. Let $A, B \in \operatorname{PFMS}(X)$ and $\alpha, \beta, \alpha_{1}, \alpha_{2}, \beta_{1}, \beta_{2} \in[0,1]$. Then we have

(i) $A_{\left[\alpha_{1}, \beta_{1}\right]} \subseteq A_{\left[\alpha_{2}, \beta_{2}\right]}$ iff $\alpha_{1} \geq \alpha_{2}$ and $\beta_{1} \leq \beta_{2}$,

(ii) $A \subseteq B$ iff $A_{[\alpha, \beta]} \subseteq B_{[\alpha, \beta]}$.

Proof. (i) Let $x \in A_{\left[\alpha_{1}, \beta_{1}\right]} \Rightarrow C M_{A}(x) \geq \alpha_{1}$ and $C N_{A}(x) \leq \beta_{1}$. Since $\alpha_{1} \geq \alpha_{2} \Rightarrow C M_{A}(x) \geq \alpha_{1} \geq \alpha_{2}$. Also, $\beta_{1} \leq \beta_{2} \Rightarrow C N_{A}(x) \leq \beta_{1} \leq \beta_{2}$. Hence, $A_{\left[\alpha_{1}, \beta_{1}\right]} \subseteq A_{\left[\alpha_{2}, \beta_{2}\right]}$.

Conversely, for $A_{\left[\alpha_{1}, \beta_{1}\right]} \subseteq A_{\left[\alpha_{2}, \beta_{2}\right]}$, it is clear that $\alpha_{1} \geq \alpha_{2}$ and $\beta_{1} \leq \beta_{2}$.

(ii) We know that $A \subseteq B \Rightarrow C M_{A}(x) \leq C M_{B}(x)$ and $C N_{A}(x) \geq C N_{B}(x) \forall x \in X$. For $x \in A_{[\alpha, \beta]}$ and $x \in B_{[\alpha, \beta]} \Rightarrow C M_{B}(x) \geq C M_{A}(x) \geq \alpha$ and $C N_{B}(x) \leq C N_{A}(x) \leq \beta$. So, $A_{[\alpha, \beta]} \subseteq B_{[\alpha, \beta]}$.

The converse is straightforward.

Corollary 1. Let $A, B \in \operatorname{PFMS}(X)$ and $\alpha, \beta, \alpha_{1}, \alpha_{2}, \beta_{1}, \beta_{2} \in[0,1]$. Then the following hold.

(i) $A^{\left[\alpha_{1}, \beta_{1}\right]} \subseteq A^{\left[\alpha_{2}, \beta_{2}\right]}$ iff $\alpha_{1} \geq \alpha_{2}$ and $\beta_{1} \leq \beta_{2}$.

(ii) $A \subseteq B$ iff $A^{[\alpha, \beta]} \subseteq B^{[\alpha, \beta]}$.

Proof. It follows from Proposition 3.

Proposition 4. Let $A \in \operatorname{PFMS}(X)$. For any $\alpha_{1}, \beta_{1} \alpha_{2}, \beta_{2} \in[0,1]$ such that $\alpha_{1} \leq \alpha_{2}$ and $\beta_{1} \geq \beta_{2}$, we have

(i) $A_{\left(\alpha_{2}, \beta_{2}\right)} \subseteq A_{\left[\alpha_{2}, \beta_{2}\right]} \subseteq A_{\left(\alpha_{1}, \beta_{1}\right)}$ and

(ii) $A^{\left(\alpha_{1}, \beta_{1}\right)} \subseteq A^{\left(\alpha_{2}, \beta_{2}\right)} \subseteq A^{\left[\alpha_{2}, \beta_{2}\right]}$.

Proof. Combining Definition 19 and Remark 1, the proof follows.

Proposition 5. Let $A, B \in P F M S(X)$ and $\alpha, \beta \in[0,1]$. Then

(i) $(A \cap B)_{[\alpha, \beta]}=A_{[\alpha, \beta]} \cap B_{[\alpha, \beta]}$,

(ii) $(A \cup B)_{[\alpha, \beta]}=A_{[\alpha, \beta]} \cup B_{[\alpha, \beta]}$.

Proof. (i) If $A, B \in \operatorname{PFMS}(X) \Rightarrow A \cap B \subseteq A$ and $A \cap B \subseteq B$. By Proposition 3, $(A \cap B)_{[\alpha, \beta]} \subseteq A_{[\alpha, \beta]}$ and $(A \cap B)_{[\alpha, \beta]} \subseteq B_{[\alpha, \beta]} \Rightarrow(A \cap B)_{[\alpha, \beta]} \subseteq A_{[\alpha, \beta]} \cap B_{[\alpha, \beta]}$.

Again, suppose $x \in A_{[\alpha, \beta]} \cap B_{[\alpha, \beta]} \Rightarrow x \in A_{[\alpha, \beta]}$ and $x \in B_{[\alpha, \beta]}$, then

$$
\begin{aligned}
A_{[\alpha, \beta]} \cap B_{[\alpha, \beta]} & =\left\{x \in X \mid C M_{A}(x) \geq \alpha, C N_{A}(x) \leq \beta\right\} \cap\left\{x \in X \mid C M_{B}(x) \geq \alpha, C N_{B}(x) \leq \beta\right\} \\
& =\left\{x \in X \mid \min \left[C M_{A}(x) \geq \alpha, C M_{B}(x) \geq \alpha\right], \max \left[C N_{A}(x) \leq \beta, C N_{B}(x) \leq \beta\right]\right\} \\
& =\left\{x \in X \mid \min \left[C M_{A}(x), C M_{B}(x)\right] \geq \alpha, \max \left[C N_{A}(x), C N_{B}(x)\right] \leq \beta\right\} \\
& =\left\{x \in X \mid C M_{A \cap B}(x) \geq \alpha, C N_{A \cap B}(x) \leq \beta\right\} \\
& \subseteq(A \cap B)_{[\alpha, \beta]} .
\end{aligned}
$$

Consequently, $x \in A_{[\alpha, \beta]} \cap B_{[\alpha, \beta]} \Rightarrow x \in(A \cap B)_{[\alpha, \beta]}$. Hence $(A \cap B)_{[\alpha, \beta]}=A_{[\alpha, \beta]} \cap B_{[\alpha, \beta]}$.

(ii) For $A, B \in \operatorname{PFMS}(X)$, it is clear that $A \subseteq A \cup B$ and $B \subseteq A \cup B$. By Proposition 3, implies $A_{[\alpha, \beta]} \subseteq$ $(A \cup B)_{[\alpha, \beta]}$ and $B_{[\alpha, \beta]} \subseteq(A \cup B)_{[\alpha, \beta]}$, that is, $A_{[\alpha, \beta]} \cup B_{[\alpha, \beta]} \subseteq(A \cup B)_{[\alpha, \beta]}$.

Also, $x \in(A \cup B)_{[\alpha, \beta]} \Rightarrow C M_{(A \cup B)}(x) \geq \alpha$ and $C N_{(A \cup B)}(x) \leq \beta$, that is, 


$$
\begin{aligned}
(A \cup B)_{[\alpha, \beta]} & =\left\{x \in X \mid C M_{(A \cup B)}(x) \geq \alpha, C N_{(A \cup B)}(x) \leq \beta\right\} \\
& =\left\{x \in X \mid \max \left[C M_{A}(x), C M_{B}(x)\right] \geq \alpha, \min \left[C N_{A}(x), C N_{B}(x)\right] \leq \beta\right\} \\
& =\left\{x \in X \mid \max \left[C M_{A}(x), C M_{B}(x)\right] \geq \alpha, \min \left[C N_{A}(x), C N_{B}(x)\right] \leq \beta\right\} \\
& =\left\{x \in X \mid C M_{A}(x) \geq \alpha, C N_{A}(x) \leq \beta\right\} \cup\left\{x \in X \mid C M_{B}(x) \geq \alpha, C N_{B}(x) \leq \beta\right\} \\
& \subseteq A_{[\alpha, \beta]} \cup B_{[\alpha, \beta]} .
\end{aligned}
$$

Thus $x \in A_{[\alpha, \beta]}$ and $x \in B_{[\alpha, \beta]}$. Hence $(A \cup B)_{[\alpha, \beta]}=A_{[\alpha, \beta]} \cup B_{[\alpha, \beta]}$.

Corollary 2. Let $A, B \in P F M S(X)$ and $\alpha, \beta \in[0,1]$. Then

(i) $(A \cap B)^{[\alpha, \beta]}=A^{[\alpha, \beta]} \cap B^{[\alpha, \beta]}$,

(ii) $(A \cup B)^{[\alpha, \beta]}=A^{[\alpha, \beta]} \cup B^{[\alpha, \beta]}$.

Proof. Straightforward from Proposition 5.

Proposition 6. Suppose $\left\{A_{i}\right\}_{i \in I} \in \operatorname{PFMS}(X)$ and $\alpha, \beta \in[0,1]$, then

(i) $\left(\bigcap_{i \in I} A_{i}\right)_{[\alpha, \beta]}=\bigcap_{i \in I}\left(A_{i}\right)_{[\alpha, \beta]}$,

(ii) $\left(\bigcup_{i \in I} A_{i}\right)_{[\alpha, \beta]}=\bigcup_{i \in I}\left(A_{i}\right)_{[\alpha, \beta]}$,

(iii) $\left(\bigcap_{i \in I} A_{i}\right)^{[\alpha, \beta]}=\bigcap_{i \in I}\left(A_{i}\right)^{[\alpha, \beta]}$,

(iv) $\left(\bigcup_{i \in I} A_{i}\right)^{[\alpha, \beta]}=\bigcup_{i \in I}\left(A_{i}\right)^{[\alpha, \beta]}$.

Proof. (i) Let $C=\bigcap_{i \in I} A_{i}$, then $C M_{C}(x)=\bigwedge_{i \in I} C M_{A_{i}}(x)$ and $C N_{C}(x)=\bigvee_{i \in I} C N_{A_{i}}(x) \forall x \in X$. Thus

$$
\begin{aligned}
C_{[\alpha, \beta]} & =\left\{x \in X \mid C M_{C}(x) \geq \alpha, C N_{C}(x) \leq \beta\right\} \\
& =\left\{x \in X \mid\left(\bigwedge_{i \in I} C M_{A_{i}}(x)\right) \geq \alpha,\left(\bigvee_{i \in I} C N_{A_{i}}(x)\right) \leq \beta\right\} \\
& =\left\{x \in X \mid \bigwedge_{i \in I} C M_{A_{i}}(x) \geq \alpha, \bigvee_{i \in I} C N_{A_{i}}(x) \leq \beta\right\} \\
& =\bigcap_{i \in I}\left(A_{i}\right)_{[\alpha, \beta]} .
\end{aligned}
$$

Hence $\left(\bigcap_{i \in I} A_{i}\right)_{[\alpha, \beta]}=\bigcap_{i \in I}\left(A_{i}\right)_{[\alpha, \beta]}$.

(ii)-(iv) follow similarly.

Remark 2. Suppose $A, B, C \in P F M S(X)$ such that $B \subseteq C$. Then for $\alpha, \beta \in[0,1]$, we have

(i) $(A \cap B)_{[\alpha, \beta]} \subseteq(A \cap C)_{[\alpha, \beta]}$,

(ii) $(A \cup B)_{[\alpha, \beta]}^{[\alpha, \beta]} \subseteq(A \cup C)_{[\alpha, \beta]}$,

(iii) $(A \cap B)^{[\alpha, \beta]} \subseteq(A \cap C)^{[\alpha, \beta]}$,

(iv) $(A \cup B)^{[\alpha, \beta]} \subseteq(A \cup C)^{[\alpha, \beta]}$.

Proposition 7. Let $f$ be a function from $X$ to $Y, A \in P F M S(X)$ and $B \in P F M S(Y)$, respectively. Then, for any $\alpha, \beta \in[0,1]$, we have

(i) $f\left(A_{[\alpha, \beta]}\right) \subseteq(f(A))_{[\alpha, \beta]}$,

(ii) $f^{-1}\left(B_{[\alpha, \beta]}\right)=\left(f^{-1}(B)\right)_{[\alpha, \beta]}$,

(iii) $f\left(A_{(\alpha, \beta)}\right) \subseteq f\left(A_{[\alpha, \beta]}\right) \subseteq(f(A))_{[\alpha, \beta]}$,

(iv) $f^{-1}\left(B_{(\alpha, \beta)}\right) \subseteq f^{-1}\left(B_{[\alpha, \beta]}\right)=\left(f^{-1}(B)\right)_{[\alpha, \beta]}$. 
Proof. (i) Let $y \in f\left(A_{[\alpha, \beta]}\right)$, then $\exists x \in A_{[\alpha, \beta]}$ such that $f(x)=y$ and $C M_{A}(x) \geq \alpha, C N_{A}(x) \leq \beta$. Consequently, we get

$$
C M_{A}\left(f^{-1}(y)\right) \geq \alpha, \alpha \in[0,1] \text { implies } C M_{f(A)}(y) \geq \alpha, \alpha \in[0,1],
$$

Similarly,

$$
C N_{A}\left(f^{-1}(y)\right) \leq \beta, \beta \in[0,1] \text { implies } C N_{f(A)}(y) \leq \beta, \beta \in[0,1],
$$

and so, $y \in(f(A))_{[\alpha, \beta]}$. Hence, $f\left(A_{[\alpha]}\right) \subseteq(f(A))_{[\alpha]}$.

(ii) For every $x, x \in f^{-1}\left(B_{[\alpha, \beta]}\right) \Leftrightarrow f(x) \in B_{[\alpha, \beta]} \Leftrightarrow C M_{B}(f(x)) \geq \alpha$ and $C N_{B}(f(x)) \leq \beta$. Thus $C M_{f^{-1}(B)}(x)=C M_{B}(f(x)) \geq \alpha$ and $C N_{f^{-1}(B)}(x)=C N_{B}(f(x)) \leq \beta$ that is, $x \in\left(f^{-1}(B)\right)_{[\alpha, \beta]}$. Hence, $f^{-1}\left(B_{[\alpha, \beta]}\right)=\left(f^{-1}(B)\right)_{[\alpha, \beta]}$.

(iii) Since $A_{(\alpha, \beta)} \subseteq A_{[\alpha, \beta]}$, then $f\left(A_{(\alpha, \beta)}\right) \subseteq f\left(A_{[\alpha, \beta]}\right)$. Hence, the result follows from (i).

(iv) Also, $B_{(\alpha, \beta)} \subseteq B_{[\alpha, \beta]}$ and so, $f^{-1}\left(A_{(\alpha, \beta)}\right) \subseteq f^{-1}\left(A_{[\alpha, \beta]}\right)$ by the same reasons as in (iii). The proof is completed by (ii).

Corollary 3. Suppose $f$ is a function from $X$ to $Y$. If $A \in P F M S(X)$ and $B \in P F M S(Y)$, respectively, then for at least one $\alpha, \beta \in[0,1]$,

(i) $f\left(A^{[\alpha, \beta]}\right) \subseteq(f(A))^{[\alpha, \beta]}$,

(ii) $f^{-1}\left(B^{[\alpha, \beta]}\right)=\left(f^{-1}(B)\right)^{[\alpha, \beta]}$,

(iii) $f\left(A^{(\alpha, \beta)}\right) \subseteq f\left(A^{[\alpha, \beta]}\right) \subseteq(f(A))^{[\alpha, \beta]}$,

(iv) $f^{-1}\left(B^{(\alpha, \beta)}\right) \subseteq f^{-1}\left(B^{[\alpha, \beta]}\right)=\left(f^{-1}(B)\right)^{[\alpha, \beta]}$.

Proof. Similar to Proposition 7.

\subsubsection{Some modal operators on PFMS}

Now, we propose and explicate some modal operators on PFMS, which transform every PFMS to fuzzy multiset. These modal operators are similar to the operators necessity and possibility defined in some modal logics.

Definition 20. Let $A \in \operatorname{PFMS}(X)$. Then we define the following operators:

(i) the necessity operator

$$
\square A=\left\{\left\langle x, C M_{A}(x), \sqrt{1-\left(C M_{A}(x)\right)^{2}}\right\rangle \mid x \in X\right\}
$$

(ii) the possibility operator

$$
\diamond A=\left\{\left\langle x, \sqrt{1-\left(C N_{A}(x)\right)^{2}}, C N_{A}(x)\right\rangle \mid x \in X\right\} .
$$

Remark 3. If $A$ is an ordinary fuzzy multiset, then $\square A=A=\diamond A$. An ordinary fuzzy multiset $A$ can also be written in PFMS setting as

$$
A=\left\{\left\langle x, C M_{A}(x), \sqrt{1-\left(C M_{A}(x)\right)^{2}}\right\rangle \mid x \in X\right\}
$$

or

$$
A=\left\{\left\langle x, \sqrt{1-\left(\mathrm{CN}_{A}(x)\right)^{2}}, \mathrm{CN}_{A}(x)\right\rangle \mid x \in X\right\} .
$$

Theorem 21. Let $A \in \operatorname{PFMS}(X)$. Then the following properties hold:

(i) $\overline{\square \bar{A}}=\diamond A$

(ii) $\overline{\nabla \bar{A}}=\square A$ 
(iii) $\square \square A=\square A$

(iv) $\diamond \diamond A=\diamond A$

(v) $\square \diamond A=\diamond A$

(vi) $\diamond \square A=\square A$.

Proof. Let $x \in X$. Using Definition 20, we have

(i)

$$
\begin{aligned}
\overline{\square \bar{A}} & =\overline{\square \overline{\left.\left\langle\left\langle x, C M_{A}(x), C N_{A}(x)\right\rangle\right| x \in X\right\}}} \\
& =\overline{\square\left\{\left\langle x, C N_{A}(x), C M_{A}(x)\right\rangle \mid x \in X\right\}} \\
& =\left\{\left\langle x, C N_{A}(x), \sqrt{1-\left(C N_{A}(x)\right)^{2}}\right\rangle \mid x \in X\right\} \\
& =\left\{\left\langle x, \sqrt{1-\left(C N_{A}(x)\right)^{2}}, C N_{A}(x)\right\rangle \mid x \in X\right\} \\
& =\diamond A .
\end{aligned}
$$

(ii)

$$
\begin{aligned}
\overline{\nabla \bar{A}} & =\overline{\nabla \overline{\left\{\left\langle x, C M_{A}(x), C N_{A}(x)\right\rangle \mid x \in X\right\}}} \\
& =\overline{\nabla\left\{\left\langle x, C N_{A}(x), C M_{A}(x)\right\rangle \mid x \in X\right\}} \\
& =\left\{\left\langle x, \sqrt{1-\left(C M_{A}(x)\right)^{2}}, C M_{A}(x)\right\rangle \mid x \in X\right\} \\
& =\left\{\left\langle x, C M_{A}(x), \sqrt{1-\left(C M_{A}(x)\right)^{2}}\right\rangle \mid x \in X\right\} \\
& =\square A .
\end{aligned}
$$

(iii)

$$
\begin{aligned}
\square \square A & =\square \square\left\{\left\langle x, C M_{A}(x), C N_{A}(x)\right\rangle \mid x \in X\right\} \\
& =\square\left\{\left\langle x, C M_{A}(x), \sqrt{1-\left(C M_{A}(x)\right)^{2}}\right\rangle \mid x \in X\right\} \\
& =\left\{\left\langle x, C M_{A}(x), \sqrt{1-\left(C M_{A}(x)\right)^{2}}\right\rangle \mid x \in X\right\} \\
& =\square A .
\end{aligned}
$$

(iv)

$$
\begin{aligned}
\diamond \diamond A & =\diamond \diamond\left\{\left\langle x, C M_{A}(x), C N_{A}(x)\right\rangle \mid x \in X\right\} \\
& =\diamond\left\{\left\langle x, \sqrt{1-\left(C N_{A}(x)\right)^{2}}, C N_{A}(x)\right\rangle \mid x \in X\right\} \\
& =\left\{\left\langle x, \sqrt{1-\left(C N_{A}(x)\right)^{2}}, C N_{A}(x)\right\rangle \mid x \in X\right\} \\
& =\diamond A .
\end{aligned}
$$

(v)

$$
\begin{aligned}
\square \diamond A & =\square \diamond\left\{\left\langle x, C M_{A}(x), C N_{A}(x)\right\rangle \mid x \in X\right\} \\
& =\square\left\{\left\langle x, \sqrt{1-\left(C N_{A}(x)\right)^{2}}, C N_{A}(x)\right\rangle \mid x \in X\right\} \\
& =\left\{\left\langle x, \sqrt{1-\left(C N_{A}(x)\right)^{2}}, \sqrt{1-\left(\sqrt{\left.1-\left(C N_{A}(x)\right)^{2}\right)}\right)^{2}}\right\rangle \mid x \in X\right\} \\
& =\left\{\left\langle x, \sqrt{1-\left(C N_{A}(x)\right)^{2}}, \sqrt{1-\left(1-\left(C N_{A}(x)\right)^{2}\right)}\right\rangle \mid x \in X\right\}
\end{aligned}
$$




$$
\begin{aligned}
& =\left\{\left\langle x, \sqrt{1-\left(C N_{A}(x)\right)^{2}}, \sqrt{\left.\left(C N_{A}(x)\right)^{2}\right)}\right\rangle \mid x \in X\right\} \\
& =\left\{\left\langle x, \sqrt{1-\left(C N_{A}(x)\right)^{2}}, C N_{A}(x)\right\rangle \mid x \in X\right\} \\
& =\diamond A .
\end{aligned}
$$

(vi)

$$
\begin{aligned}
\diamond \square A & =\diamond \square\left\{\left\langle x, C M_{A}(x), C N_{A}(x)\right\rangle \mid x \in X\right\} \\
& \left.=\diamond\left\{\left\langle x, C M_{A}(x)\right), \sqrt{1-\left(C M_{A}(x)\right)^{2}}\right\rangle \mid x \in X\right\} \\
& =\left\{\left\langle x, \sqrt{1-\left(\sqrt{\left.1-\left(C M_{A}(x)\right)^{2}\right)}\right)^{2}}, \sqrt{1-\left(C M_{A}(x)\right)^{2}}\right\rangle \mid x \in X\right\} \\
& =\left\{\left\langle x, \sqrt{1-\left(1-\left(C M_{A}(x)\right)^{2}\right)}, \sqrt{1-\left(C M_{A}(x)\right)^{2}}\right\rangle \mid x \in X\right\} \\
& =\left\{\left\langle x, \sqrt{\left(C M_{A}(x)\right)^{2}}, \sqrt{\left.1-\left(C M_{A}(x)\right)^{2}\right)}\right\rangle \mid x \in X\right\} \\
& =\left\{\left\langle x, C M_{A}(x), \sqrt{1-\left(C M_{A}(x)\right)^{2}}\right\rangle \mid x \in X\right\} \\
& =\square A .
\end{aligned}
$$

Corollary 4. Let $A \in \operatorname{PFMS}(X)$. Then the following properties hold:

(i) $\overline{\square \bar{A}}=\square \diamond A=\diamond \diamond A$

(ii) $\overline{\diamond \bar{A}}=\diamond \square A=\square \square A$.

Proof. Straightforward from Theorem 21.

Theorem 22. Let $A, B \in P F M S(X)$. Then the following properties hold:
(i) $\square(A \cap B)=\square A \cap \square B$
(ii) $\diamond(A \cap B)=\diamond A \cap \diamond B$
(iii) $\square(A \cup B)=\square A \cup \square B$
(iv) $\diamond(A \cup B)=\diamond A \cup \diamond B$.

Proof. The results are straightforward from Definitions 11 and 20, so we omit the proofs.

Theorem 23. Let $A, B \in P F M S(X)$. Then the following properties hold:
(i) $\overline{\overline{\square(A \cap B)}}=\diamond(A \cap B)$
(ii) $\overline{\nabla(A \cap B)}=\square(A \cap B)$
(iii) $\overline{\square \overline{(A \cup B)}}=\diamond(A \cup B)$
(iv) $\overline{\nabla \overline{(A \cup B)}}=\square(A \cup B)$
(v) $\square \square(A \cap B)=\square(A \cap B)$
(vi) $\diamond \diamond(A \cap B)=\diamond(A \cap B)$
(vii) $\square \square(A \cup B)=\square(A \cup B)$
(viii) $\diamond \diamond(A \cup B)=\diamond(A \cup B)$
(ix) $\square \diamond(A \cap B)=\diamond(A \cap B)$
(x) $\diamond \square(A \cap B)=\square(A \cap B)$
(xi) $\square \diamond(A \cup B)=\diamond(A \cup B)$
(xii) $\diamond \square(A \cup B)=\square(A \cup B)$.

Proof. Synthesizing Theorems 21 and 22, the proofs follow. 
Corollary 5. Let $A, B \in P F M S(X)$. Then the following properties hold:

(i) $\overline{\overline{\square(A \cap B)}}=\diamond \diamond(A \cap B)=\square \diamond(A \cap B)$

(ii) $\overline{\overline{\triangle(A \cap B)}}=\square \square(A \cap B)=\diamond \square(A \cap B)$

(iii) $\overline{\square \overline{(A \cup B)}}=\diamond \diamond(A \cup B)=\square \diamond(A \cup B)$

(iv) $\overline{\nabla \overline{(A \cup B)}}=\square \square(A \cup B)=\diamond \square(A \cup B)$.

Proof. Straightforward from Theorem 23.

Theorem 24. Let $A \in \operatorname{PFMS}(X)$. Then $\square A \subset A \subset \diamond A$.

Proof. Recall that for $A=\left\{\left\langle x, C M_{A}(x), C N_{A}(x)\right\rangle \mid x \in X\right\}$, we have

$$
\square A=\left\{\left\langle x, C M_{A}(x), \sqrt{1-\left(C M_{A}(x)\right)^{2}}\right\rangle \mid x \in X\right\}
$$

and

$$
\diamond A=\left\{\left\langle x, \sqrt{1-\left(C N_{A}(x)\right)^{2}}, C N_{A}(x)\right\rangle \mid x \in X\right\} .
$$

Also, $A \subset B \Leftrightarrow A \subseteq B$ and $A \neq B$, and $A \subseteq B \Leftrightarrow C M_{A}(x) \leq C M_{B}(x)$ and either $C N_{A}(x) \geq C N_{B}(x)$ (or $\left.C N_{A}(x) \leq C N_{B}(x)\right) \forall x \in X$.

To prove that $\square A \subset A$, it is sufficient to show that

$$
\sqrt{1-\left(C M_{A}(x)\right)^{2}} \geq C N_{A}(x) .
$$

From Definition 6, we have

$$
\begin{aligned}
\left(C M_{A}(x)\right)^{2}+\left(C N_{A}(x)\right)^{2} \leq 1 & \Rightarrow\left(C N_{A}(x)\right)^{2} \leq 1-\left(C M_{A}(x)\right)^{2} \\
& \Rightarrow C N_{A}(x) \leq \sqrt{1-\left(C M_{A}(x)\right)^{2}}
\end{aligned}
$$

that is $\sqrt{1-\left(C M_{A}(x)\right)^{2}} \geq C N_{A}(x) \forall x \in X$. Thus $\square A \subset A$.

Again, we show that $A \subset \diamond A$. To see this, it is enough to prove that

$$
C M_{A}(x) \leq \sqrt{1-\left(C N_{A}(x)\right)^{2}} .
$$

By Definition 6, We get

$$
\begin{aligned}
\left(C M_{A}(x)\right)^{2}+\left(C N_{A}(x)\right)^{2} \leq 1 & \Rightarrow\left(C M_{A}(x)\right)^{2} \leq 1-\left(C N_{A}(x)\right)^{2} \\
& \Rightarrow C M_{A}(x) \leq \sqrt{1-\left(C N_{A}(x)\right)^{2}} \forall x \in X .
\end{aligned}
$$

Hence, $A \subset \diamond A$, and the proof is complete.

\section{Composite relation on PFMS and its application in course placements}

\subsection{Composite relation defined on PFMS}

In what follows, we define the composite relation on PFMS.

Definition 25. Let $X$ and $Y$ be two non-empty sets. A Pythagorean fuzzy multi-relation (PFMR), $R$ from $X$ to $Y$ is a PFMS of $X \times Y$ characterised by the count membership function, $C M_{R}$ and count non-membership function, $C N_{R}$. A PF multi-relation or PFMR from $X$ to $Y$ is denoted by $R(X \rightarrow Y)$.

Definition 26. Let $A \in P F M S(X)$. Then the max-min-max composition of $R(X \rightarrow Y)$ with $A$ is a PFMS $B$ of $Y$ denoted by $B=R \circ A$, such that its count membership and count non-membership functions are defined by 


$$
\mathrm{CM}_{B}(y)=\bigvee_{x}\left(\min \left[\mathrm{CM}_{A}(x), \mathrm{CM}_{R}(x, y)\right]\right)
$$

and

$$
\mathrm{CN}_{B}(y)=\bigwedge_{x}\left(\max \left[\mathrm{CN}_{A}(x), \mathrm{CN}_{R}(x, y)\right]\right)
$$

$\forall x \in X$ and $y \in Y$, where $\bigvee=$ maximum, $\Lambda=$ minimum.

Definition 27. Let $Q(X \rightarrow Y)$ and $R(Y \rightarrow Z)$ be two PFMRs. Then the max-min-max composition $R \circ Q$ is a PFMR from $X$ to $Z$ such that its count membership and count non-membership functions are defined by

$$
C M_{R \circ Q}(x, z)=\bigvee_{y}\left(\min \left[C M_{Q}(x, y), C M_{R}(y, z)\right]\right)
$$

and

$$
C N_{R \circ Q}(x, z)=\bigwedge_{y}\left(\max \left[C N_{Q}(x, y), C N_{R}(y, z)\right]\right)
$$

$\forall(x, z) \in X \times Z$ and $\forall y \in Y$.

Remark 4. From Definitions 26 and 27, the max-min-max composition $B$ or $R \circ Q$ is calculated by

$$
B=C M_{B}(y)-C N_{B}(y) C H_{B}(y)
$$

$\forall y \in Y$ or

$$
R \circ Q=C M_{R \circ Q}(x, z)-C N_{R \circ Q}(x, z) C H_{R \circ Q}(x, z)
$$

$\forall(x, z) \in X \times Z$

Proposition 8. If $R$ and $S$ are two PFMRs on $X \times Y$ and $Y \times Z$, respectively. Then

(i) $\left(R^{-1}\right)^{-1}=R$,

(ii) $(S \circ R)^{-1}=R^{-1} \circ S^{-1}$.

\subsection{Composite relation on PFMS in course placement}

We apply the notion of PFMR as follows. Let

$$
S=\left\{s_{1}, \ldots, s_{l}\right\}, C=\left\{c_{1}, \ldots, c_{m}\right\} \text { and } A=\left\{a_{1}, \ldots, a_{n}\right\}
$$

be finite set of subjects related to the courses, finite set of courses and finite set of applicants, respectively. Assume there are two PFMRs, $R(A \rightarrow S)$ and $U(S \rightarrow C)$ such that

$$
R=\left\{\left\langle(a, s), C M_{R}(a, s), C N_{R}(a, s)\right\rangle \mid(a, s) \in A \times S\right\}
$$

and

$$
U=\left\{\left\langle(s, c), C M_{U}(s, c), C N_{U}(s, c)\right\rangle \mid(s, c) \in S \times C\right\},
$$

where $C M_{R}(a, s)$ signifies the grade to which the applicant, a passes the related subject requirement, $s$, and $C N_{R}(a, s)$ signifies the grade to which the applicant, $a$ does not pass the related subject requirement, $s$.

Similarly, $C M_{U}(s, c)$ signifies the grade to which the related subject requirement, $s$ determines the course, $c$, and $C N_{U}(s, c)$ signifies the grade to which the related subject requirement, $s$ does not determine the course, c.

The composition, $T$ of $R$ and $U$ is given as $T=R \circ U$. This describes the state in which the applicants, $a_{i}$ with respect to the related subjects requirement, $s_{j}$ fit the courses, $c_{k}$. Thus:

$$
C M_{T}\left(a_{i}, c_{k}\right)=\bigvee_{s_{j} \in S}\left\{\min \left[C M_{R}\left(a_{i}, s_{j}\right), C M_{U}\left(s_{j}, c_{k}\right)\right]\right\}
$$


and

$$
C N_{T}\left(a_{i}, c_{k}\right)=\bigwedge_{s_{j} \in S}\left\{\max \left[C N_{R}\left(a_{i}, s_{j}\right), C N_{U}\left(s_{j}, c_{k}\right)\right]\right\}
$$

$\forall a_{i} \in A$ and $c_{k} \in C$, where $i, j$ and $k$ take values from $1, \ldots, n$. The career placement can be attained if the value of $T$ given by

$$
T=C M_{T}\left(a_{i}, c_{k}\right)-C N_{T}\left(a_{i}, c_{k}\right) C H_{T}\left(a_{i}, c_{k}\right),
$$

as calculated from $R$ and $U$ for the placements of $a_{i}$ into any $c_{k}$ with respect to $s_{j}$ is the greatest, greater than 0.5 .

\subsubsection{Case study}

Let $A=\{$ Eli, Ella, Avi, Joe, Jones $\}$ be the set of applicants for the course placements,

$$
C=\{\text { medicine, pharmacy, surgery, anatomy, physiology }\}
$$

be the set of courses the applicants are competing for, and

$$
S=\{\text { English Lang., Maths, Biology, Physics, Chemistry, Health Sci. }\}
$$

be the set of subjects' requirement to the set of courses.

Suppose the PFMR, $R(A \rightarrow S)$ is given in Table 3. This data in Pythagorean fuzzy multi-values are supposedly drawn after the applicants sat for a multiple choice qualification assessments on the listed subjects within a specified time, for two different times where the first and second assessments are closely related to checkmate the effect of test contingencies.

The first entries are the membership values signifying the Pythagorean fuzzy multi-values of the marks allocated to the questions the applicants answered, and the second entries are the non-membership values signifying the Pythagorean fuzzy multi-values of the marks allocated to the questions failed. Converting the PFMSs in Table 2 to PFSs for easy computation, the results in Table 3 are obtained.

Table 2. $R(A \rightarrow S)$

\begin{tabular}{||l|l|l|l|l|l|l||}
\hline \hline$R$ & English & Maths & Biology & Physics & Chemistry & Health \\
\hline \hline Eli & $\langle 0.6,0.3\rangle$ & $\langle 0.5,0.4\rangle$ & $\langle 0.6,0.3\rangle$ & $\langle 0.5,0.3\rangle$ & $\langle 0.5,0.5\rangle$ & $\langle 0.6,0.2\rangle$ \\
& $\langle 0.5,0.3\rangle$ & $\langle 0.7,0.2\rangle$ & $\langle 0.6,0.3\rangle$ & $\langle 0.5,0.4\rangle$ & $\langle 0.5,0.3\rangle$ & $\langle 0.5,0.1\rangle$ \\
Ella & $\langle 0.5,0.3\rangle$ & $\langle 0.6,0.3\rangle$ & $\langle 0.5,0.3\rangle$ & $\langle 0.4,0.5\rangle$ & $\langle 0.7,0.2\rangle$ & $\langle 0.7,0.1\rangle$ \\
& $\langle 0.4,0.3\rangle$ & $\langle 0.8,0.2\rangle$ & $\langle 0.7,0.3\rangle$ & $\langle 0.6,0.2\rangle$ & $\langle 0.7,0.2\rangle$ & $\langle 0.7,0.3\rangle$ \\
Avi & $\langle 0.7,0.3\rangle$ & $\langle 0.7,0.2\rangle$ & $\langle 0.7,0.3\rangle$ & $\langle 0.5,0.4\rangle$ & $\langle 0.4,0.5\rangle$ & $\langle 0.6,0.3\rangle$ \\
& $\langle 0.6,0.2\rangle$ & $\langle 0.7,0.2\rangle$ & $\langle 0.5,0.2\rangle$ & $\langle 0.4,0.5\rangle$ & $\langle 0.5,0.5\rangle$ & $\langle 0.7,0.3\rangle$ \\
Joe & $\langle 0.6,0.4\rangle$ & $\langle 0.8,0.2\rangle$ & $\langle 0.6,0.3\rangle$ & $\langle 0.6,0.3\rangle$ & $\langle 0.6,0.3\rangle$ & $\langle 0.7,0.2\rangle$ \\
& $\langle 0.6,0.3\rangle$ & $\langle 0.6,0.1\rangle$ & $\langle 0.6,0.3\rangle$ & $\langle 0.5,0.2\rangle$ & $\langle 0.7,0.2\rangle$ & $\langle 0.7,0.2\rangle$ \\
Jones & $\langle 0.8,0.1\rangle$ & $\langle 0.7,0.2\rangle$ & $\langle 0.8,0.2\rangle$ & $\langle 0.7,0.1\rangle$ & $\langle 0.6,0.1\rangle$ & $\langle 0.8,0.1\rangle$ \\
& $\langle 0.6,0.2\rangle$ & $\langle 0.5,0.1\rangle$ & $\langle 0.5,0.4\rangle$ & $\langle 0.7,0.1\rangle$ & $\langle 0.4,0.2\rangle$ & $\langle 0.8,0.1\rangle$ \\
\hline \hline
\end{tabular}

Table 3. $R(A \rightarrow S)$

\begin{tabular}{||l|l|l|l|l|l|l||}
\hline \hline$R$ & English & Maths & Biology & Physics & Chemistry & Health \\
\hline \hline Eli & $\langle 0.55,0.30\rangle$ & $\langle 0.60,0.30\rangle$ & $\langle 0.60,0.30\rangle$ & $\langle 0.50,0.35\rangle$ & $\langle 0.50,0.40\rangle$ & $\langle 0.55,0.15\rangle$ \\
Ella & $\langle 0.45,0.30\rangle$ & $\langle 0.70,0.25\rangle$ & $\langle 0.60,0.30\rangle$ & $\langle 0.50,0.35\rangle$ & $\langle 0.70,0.20\rangle$ & $\langle 0.70,0.20\rangle$ \\
Avi & $\langle 0.65,0.25\rangle$ & $\langle 0.70,0.20\rangle$ & $\langle 0.60,0.25\rangle$ & $\langle 0.45,0.45\rangle$ & $\langle 0.45,0.50\rangle$ & $\langle 0.65,0.30\rangle$ \\
Joe & $\langle 0.60,0.35\rangle$ & $\langle 0.70,0.15\rangle$ & $\langle 0.60,0.30\rangle$ & $\langle 0.55,0.25\rangle$ & $\langle 0.65,0.25\rangle$ & $\langle 0.70,0.20\rangle$ \\
Jones & $\langle 0.70,0.15\rangle$ & $\langle 0.60,0.15\rangle$ & $\langle 0.65,0.30\rangle$ & $\langle 0.70,0.10\rangle$ & $\langle 0.50,0.15\rangle$ & $\langle 0.80,0.10\rangle$ \\
\hline \hline
\end{tabular}

PFMR, $U(S \rightarrow C)$ is the School bench-mark for admission into the mentioned courses in Pythagorean fuzzy values. The data is in Table 4. 
Table 4. $U(S \rightarrow C)$

\begin{tabular}{||l|l|l|l|l|l||}
\hline \hline$U$ & medicine & pharmacy & surgery & anatomy & physiology \\
\hline \hline English & $\langle 0.8,0.1\rangle$ & $\langle 0.9,0.1\rangle$ & $\langle 0.5,0.4\rangle$ & $\langle 0.7,0.3\rangle$ & $\langle 0.8,0.2\rangle$ \\
Maths & $\langle 0.7,0.2\rangle$ & $\langle 0.8,0.1\rangle$ & $\langle 0.5,0.3\rangle$ & $\langle 0.5,0.4\rangle$ & $\langle 0.5,0.3\rangle$ \\
Biology & $\langle 0.9,0.1\rangle$ & $\langle 0.8,0.2\rangle$ & $\langle 0.9,0.1\rangle$ & $\langle 0.8,0.2\rangle$ & $\langle 0.9,0.1\rangle$ \\
Physics & $\langle 0.6,0.3\rangle$ & $\langle 0.5,0.2\rangle$ & $\langle 0.5,0.4\rangle$ & $\langle 0.6,0.3\rangle$ & $\langle 0.6,0.2\rangle$ \\
Chemistry & $\langle 0.8,0.2\rangle$ & $\langle 0.7,0.2\rangle$ & $\langle 0.7,0.3\rangle$ & $\langle 0.8,0.2\rangle$ & $\langle 0.7,0.2\rangle$ \\
Health & $\langle 0.8,0.1\rangle$ & $\langle 0.8,0.2\rangle$ & $\langle 0.7,0.3\rangle$ & $\langle 0.9,0.1\rangle$ & $\langle 0.8,0.2\rangle$ \\
\hline \hline
\end{tabular}

The values of $C M_{R \circ U}\left(a_{i}, c_{k}\right)$ and $C N_{R \circ U}\left(a_{i}, c_{k}\right)$ of the composition $T=R \circ U$ follow:

Table 5. $C M_{R \circ U}\left(a_{i}, c_{k}\right)$ and $C N_{R \circ U}\left(a_{i}, c_{k}\right)$

\begin{tabular}{||l|l|l|l|l|l||}
\hline \hline$C M, C N$ & medicine & pharmacy & surgery & anatomy & physiology \\
\hline \hline Eli & $\langle 0.60,0.15\rangle$ & $\langle 0.60,0.20\rangle$ & $\langle 0.60,0.30\rangle$ & $\langle 0.60,0.15\rangle$ & $\langle 0.60,0.20\rangle$ \\
Ella & $\langle 0.70,0.20\rangle$ & $\langle 0.70,0.20\rangle$ & $\langle 0.70,0.30\rangle$ & $\langle 0.70,0.20\rangle$ & $\langle 0.70,0.20\rangle$ \\
Avi & $\langle 0.70,0.20\rangle$ & $\langle 0.70,0.20\rangle$ & $\langle 0.65,0.25\rangle$ & $\langle 0.65,0.25\rangle$ & $\langle 0.65,0.25\rangle$ \\
Joe & $\langle 0.70,0.20\rangle$ & $\langle 0.70,0.15\rangle$ & $\langle 0.70,0.30\rangle$ & $\langle 0.70,0.20\rangle$ & $\langle 0.70,0.20\rangle$ \\
Jones & $\langle 0.80,0.10\rangle$ & $\langle 0.80,0.15\rangle$ & $\langle 0.70,0.30\rangle$ & $\langle 0.80,0.10\rangle$ & $\langle 0.80,0.20\rangle$ \\
\hline \hline
\end{tabular}

After computing the values of the count of hesitation margins which signify the marks loss due to the hesitation in answering questions within the specified time, $T$ is calculated as show in Table 6.

Table 6. $\mathrm{T}=\mathrm{CM}_{\mathrm{T}}-\mathrm{CN}_{\mathrm{T}} \mathrm{CH}$

\begin{tabular}{||l|l|l|l|l|l||}
\hline \hline$T$ & medicine & pharmacy & surgery & anatomy & physiology \\
\hline \hline Eli & 0.4821 & 0.4451 & 0.3775 & 0.4821 & 0.4451 \\
Ella & 0.5629 & 0.5629 & 0.5056 & 0.5629 & 0.5629 \\
Avi & 0.5629 & 0.5629 & 0.4706 & 0.4706 & 0.4706 \\
Joe & 0.5629 & 0.5953 & 0.5056 & 0.5629 & 0.5629 \\
Jones & 0.7408 & 0.7129 & 0.5056 & 0.7408 & 0.6869 \\
\hline \hline
\end{tabular}

The course placements are carried out on the basis of which of the applicant has the greatest $T$ such that $T>0.5$. However, if an applicant is suitable to study more than one courses based on the value of $T$, then the applicant would be allowed to make a personal choice within the range of the courses he/she has the greatest $T$ such that $T>0.5$.

From Table 6, the following placements are made: Eli is not suitable to read any of the courses; Ella is suitable to read any of medicine, pharmacy, anatomy and physiology; Avi is suitable to read either medicine or pharmacy; Joe is suitable to read pharmacy and Jones is suitable to read either medicine or anatomy. Suppose there is only one slot for medicine, it would be given to Jones. Also, if one applicant is to ready pharmacy, it would be Joe. Notwithstanding, Jones is very suitable to read any of the courses ahead of all the applicants.

\section{Conclusion}

We have proposed the idea of PFMSs as the generalization of PFSs such that each of membership degree, non-membership degree and hesitation margin of PFS, are allowed to repeat as count membership degrees, count non-membership degrees and count hesitation degrees. It was shown that PFMS is either the incorporation of IFMS into PFS setting or PFS in multiset framework. We have discussed some algebraic properties of PFMS and proposed the analogs of the modal logic operators "necessity" and "possibility" with some results. Also the ideas of level sets, cuts, accuracy and score functions were established in the setting of PFMS with a number of results. The notion of composite relation is proposed in PFMSs and applied to practical decision-making problem of course placements in higher institution. PFMS as a soft computing technique can find expression in image enhancement techniques for better image quality, other multi-criteria 
decision-making $(\mathrm{MCDM})$ problems or multi-attribute decision-making (MADM) problems among other potential applications.

Acknowledgments: The author would like to thank the Editor -in-chief for his technical comments and the reviewers for their insightful contributions.

Conflicts of Interest: “The author declares no conflict of interest."

\section{References}

[1] Ladeh, L. A. (1965). Fuzzy sets. Information and Control, 8, 38-353.

[2] Yager, R. R. (1986). On the theory of bags. International Journal of General Systems, 13, 23-37.

[3] Syropoulos, A. (2001). Mathematics of multisets. Springer-Verlag Berlin Heidelberg, 347-358.

[4] Miyamoto, S. (1996). Basic operations of fuzzy multisets. Journal of Japan Society of Fuzzy Theory and Systems, 8(4), 639-645.

[5] Atanassov, K. T. (1983). Intuitionistic fuzzy sets. VII ITKR's Session, Sofia.

[6] Atanassov, K. T. (1986). Intuitionistic fuzzy sets. Fuzzy Sets and Systems, 20, 87-96.

[7] Atanassov, K. T. (1989). Geometrical interpretation of the elements of the intuitionistic fuzzy objects. Preprint IM-MFAIS-1-89, Sofia.

[8] Atanassov, K. T. (1999). Intuitionistic fuzzy sets: theory and applications. Physica-Verlag, Heidelberg.

[9] Chen, S. M., Cheng, S. H. \& Chiou, C. H. (2016). Fuzzy multiattribute group decision making based on intuitionistic fuzzy sets and evidential reasoning methodology. Information Fusion, 27, 215-227.

[10] De, S. K., Biswas, R. \& Roy, A. R. (2001). An application of intuitionistic fuzzy sets in medical diagnosis. Fuzzy Sets and Systems, 117(2), 209-213.

[11] Ejegwa, P. A., Akubo, A. J. \& Joshua, O. M. (2014). Intuitionistic fuzzy sets in career determination. Journal of Information and Computing Science, 9(4), 285-288.

[12] Garg, H. \& Kumar, K. (2018). An advance study on the similarity measures of intuitionistic fuzzy sets based on the set pair analysis theory and their application in decision making. Soft Computing, 22(15), 4959-4970.

[13] Szmidt, E. \& Kacprzyk, J. (2001). Intuitionistic fuzzy sets in some medical applications. Note IFS, 7(4), 58-64.

[14] Yager, R. R. (2013). Pythagorean membership grades in multicriteria decision making. Technical Report MII-3301 Machine Intelligence Institute, Iona College, New Rochelle, NY.

[15] Zhang, X. (2016). A novel approach based on similarity measure for Pythagorean fuzzy multiple criteria group decision making. International Journal of Intelligent Systems, 31, 593-661.

[16] Shinoj, T. K. \& Sunil, J. J. (2012). Intuitionistic fuzzy multisets and its application in medical diagnosis. International Journal of Mathematical and Computational Science, 6, 34-38.

[17] Ejegwa, P. A. (2015). Mathematical techniques to transform intuitionistic fuzzy multisets to fuzzy sets. Journal of Information and Computing Science, 10(2), 169-172.

[18] Ejegwa, P. A. (2016). Some operations on intuitionistic fuzzy multisets. Journal of Fuzzy Mathematics, 24(4), 761-768.

[19] Shinoj, T. K. \& Sunil, J. J. (2013). Accuracy in collaborative robotics: an intuitionistic fuzzy multiset approach. Global Journal of Science Frontier Research: Mathematics and Decision Science, 13, 21-28.

[20] Das, S., Kar, M. B. \& Kar, S. (2013). Group multi-criteria decision making using intuitionistic multi-fuzzy sets. Journal of Uncertainty Analysis and Applications, 1(10), doi:10.1186/2195-5468-1-10.

[21] Rajarajeswari, P. \& Uma, N. (2013). Hausdroff similarity measures for intuitionistic fuzzy multisets and its application in medical diagnosis. International Journal of Mathematical Archive, 4(10), 106-111.

[22] Ejegwa, P. A. \& Awolola, J. A. (2014). Intuitionistic fuzzy multisets in binomial distributions. International Journal of Scientific and Technology Research, 3(4), 335-337.

[23] Rajarajeswari, P. \& Uma, N. (2014). Normalized Hamming measure for intuitionistic fuzzy multisets and its application in medical diagnosis. International Journal of Mathematics Trends and Technology, 5(3), 219-225.

[24] Ejegwa, P. A. (2016). On intuitionistic fuzzy multisets theory and its application in diagnostic medicine. MAYFEB Journal of Mathematics, 4, 13-22.

[25] Ejegwa, P. A., Kwarkar, L. N. \& Ihuoma, K. N. (2016). Application of intuitionistic fuzzy multisets in appointment process. International Journal of Computer Applications, 135(1), 1-4.

[26] Ejegwa, P. A., Tyongi, B. B., Osuwa, S. C. \& Atime, A. A. (2017). Career determination using intuitionistic fuzzy multisets approach. MAYFEB Journal of Mathematics, 1, 1-10.

[27] Ulucay, V., Deli, I. \& Sahin, M. (2019). Intuitionistic trapezoidal fuzzy multi-numbers and its application to multi-criteria decision-making problems. Complex and Intelligent Systems, 5, 65-78. 
(C) 2020 by the author; licensee PSRP, Lahore, Pakistan. This article is an open access article distributed under the terms and conditions of the Creative Commons Attribution (CC-BY) license (http://creativecommons.org/licenses/by/4.0/). 